\title{
BPS states, knots, and quivers
}

\author{
Piotr Kucharski, ${ }^{1}$ Markus Reineke, ${ }^{2}$ Marko Stošić, ${ }^{3,4}$ and Piotr Sułkowski ${ }^{1,5}$ \\ ${ }^{1}$ Faculty of Physics, University of Warsaw, Pasteura 5, 02-093 Warsaw, Poland \\ ${ }^{2}$ Faculty of Mathematics, Ruhr-Universität Bochum, Universitätsstrasse 150, 44780 Bochum, Germany \\ ${ }^{3}$ Departamento de Matemática, Instituto Superior Técnico, \\ CAMGSD, Avenida Rovisco Pais, 1049-001 Lisboa, Portugal \\ ${ }^{4}$ Mathematical Institute SANU, Knez Mihailova 36, 11000 Beograd, Serbia \\ ${ }^{5}$ Walter Burke Institute for Theoretical Physics, California Institute of Technology, \\ Pasadena, California 91125, USA
}

(Received 1 March 2017; published 27 December 2017)

\begin{abstract}
We argue how to identify the supersymmetric quiver quantum mechanics description of BPS states, which arise in string theory in brane systems representing knots. This leads to a surprising relation between knots and quivers: to a given knot, we associate a quiver, so that various types of knot invariants are expressed in terms of characteristics of a moduli space of representations of the corresponding quiver. This statement can be regarded as a novel type of categorification of knot invariants, and among its various consequences we find that Labastida-Mariño-Ooguri-Vafa (LMOV) invariants of a knot can be expressed in terms of motivic Donaldson-Thomas invariants of the corresponding quiver; this proves integrality of LMOV invariants (once the corresponding quiver is identified), conjectured originally based on string theory and M-theory arguments.
\end{abstract}

DOI: $10.1103 /$ PhysRevD.96.121902

\section{INTRODUCTION}

In last few decades many intricate links between high energy physics and contemporary mathematics have been found. These links not only helped to solve some specific problems, but also led to discovery of deep and earlier unforeseen relations between different branches of mathematics. In this paper, we present a new chain of connections that in a similar vein relates a physical system-of appropriately engineered branes in string theory-with mathematical knot theory on one hand and quiver representation theory on the other hand, thereby revealing deep links between these theories.

It is particularly interesting to determine exact results in string theory. One source of such results is topological string amplitudes. Mathematically they encode GromovWitten invariants, and from a physics perspective they capture degeneracies of BPS states formed by D-branes. In case of closed string theory, these degeneracies are referred to as Gopakumar-Vafa invariants and are also conjecturally related to Donaldson-Thomas invariants of underlying Calabi-Yau manifolds [1-3].

Analogous conjectures have been formulated for systems with additional branes. Mathematically they encode open Gromov-Witten invariants, and physically they capture degeneracies of D-branes with boundaries. Furthermore, it has been conjectured that, in a system with an appropriate choice of Calabi-Yau manifold and brane configuration, such open amplitudes encode knot invariants, in particular, colored HOMFLY-PT polynomials [4-6]. This relation is a nontrivial consequence of famous relations between knot invariants, Chern-Simons gauge theory, and topological string theory $[7,8]$. This relation also predicts, thatanalogously to the closed string case-colored HOMFLYPT polynomials are encoded in BPS invariants, referred to as Labastida-Mariño-Ooguri-Vafa (LMOV) invariants (or Ooguri-Vafa invariants) [4-6]. Integrality of these invariants is an important statement for both string theory and knot theory, however, it has been verified only in very specific cases, e.g., in $[4-6,9,10]$, and more recently for some infinite families of knots and representations [11,12]. An attempt to prove this conjecture-which, however, raised some criticism-has been made in [13].

By standard arguments $[4,14,15]$, BPS states in brane systems - in particular those describing knots - should have a description in terms of supersymmetric quantum mechanics on their world volume, which, however, has not been identified to date. In this paper, we argue that such a description actually involves quiver quantum mechanics, and we identify relevant quivers. To this end, we also recall that, in another line of research, it was argued that BPS states in string theory form algebras [16]. More recently, it has been postulated that such algebras can be identified with cohomological Hall algebras [17], and the associated theory of wall crossing and motivic Donaldson-Thomas (DT) invariants turned out to play an important role in supersymmetric gauge theories and string theory. From a mathematical perspective, these ideas can be naturally implemented in the framework of quiver representations and their moduli spaces [17-19].

In this paper, we argue that BPS states enumerated by motivic Donaldson-Thomas invariants of quiver moduli spaces should be identified with those arising in the 
KUCHARSKI, REINEKE, STOŠIĆ, and SUŁKOWSKI

TABLE I. Identification of quantities associated with knots and quivers.

\begin{tabular}{ll}
\hline \hline Knots & Quivers \\
\hline Homological degrees, framing & Number of arrows and loops \\
Colored HOMFLY-PT & Motivic generating series \\
LMOV invariants & Motivic DT invariants \\
Classical LMOV invariants & Numerical DT invariants \\
Algebra of BPS states & Cohomological Hall algebra \\
\hline \hline
\end{tabular}

supersymmetric quiver quantum mechanics description of brane systems encoding knots. This observation has farreaching consequences: it leads to new ways of interpreting and computing BPS numbers and to the (idea of the) proof of the famous LMOV conjecture (and the actual proof for a large class of knots, for which the corresponding quivers are identified). From a mathematical perspective, we find direct, unexpected relations between knots and quiver representation theory, which, in particular, lead to a novel categorification of knot invariants.

In more detail, we claim that, to each knot, one can associate a quiver, for which the moduli space of representations encodes various types of knot invariants, including colored HOMFLY-PT polynomials, homological invariants, etc. In particular, LMOV invariants of a given knot can be expressed in terms of motivic Donaldson-Thomas invariants of the corresponding quiver, and integrality of the latter implies integrality of the former ones, which is how the proof of the LMOV conjecture follows. Identification of some quantities on both sides of the "knots-quivers" duality is summarized in Table I. As an example, a quiver corresponding to the trefoil knot is shown in Fig. 1.

\section{KNOT INVARIANTS, KNOT HOMOLOGIES, AND LMOV CONJECTURE}

Ever since its birth, knot theory has attracted the attention of physicists, and various knot invariants turned out to have physical interpretation. In particular, various knot polynomials have been reinterpreted as expectation values in Chern-Simons theory in [7] and, subsequently, they were expressed in terms of topological string theory amplitudes [8]. In [4], the topological string setup was related to $\mathrm{M}$ theory, and it was argued that colored HOMFLY-PT polynomials are encoded in LMOV invariants, which count
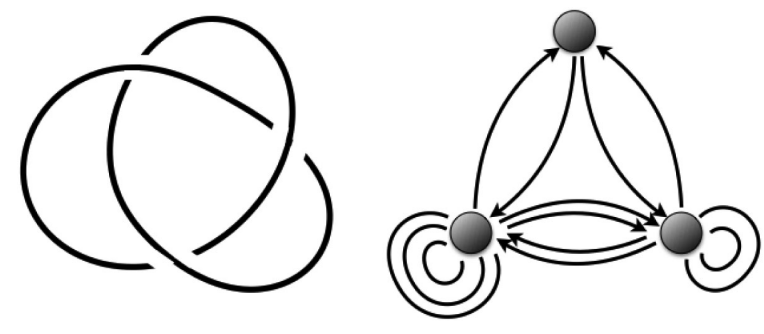

FIG. 1. Trefoil knot and the corresponding quiver.
PHYSICAL REVIEW D 96, 121902(R) (2017)

bound states of open M2-branes with M5-branes. In this work, we show, among others, how-so far conjecturalintegrality of these invariants can be proven.

Furthermore, more recent homological knot invariants, such as Khovanov homology and its more involved cousins [20-22], have been also realized in brane systems in string theory, also revealing yet to be proved new properties of those invariants [23-25]. In what follows, we show that certain information about homological knot invariants is also encoded in quiver moduli spaces.

Let us introduce first the generating function of $S^{r}$ colored HOMFLY-PT polynomials, which will be the main object of the subsequent analysis, as well as corresponding LMOV invariants. To start with, consider the Ooguri-Vafa generating function [4-6,26]

$Z(U, V)=\sum_{R} \operatorname{Tr}_{R} U \operatorname{Tr}_{R} V=\exp \left(\sum_{n=1}^{\infty} \frac{1}{n} \operatorname{Tr} U^{n} \operatorname{Tr} V^{n}\right)$,

where $U=P \exp \oint_{K} A$ is the holonomy of $U(N)$ ChernSimons gauge field along a knot $K, V$ plays a role of a source, and the sum runs over all representations $R$, i.e., all two-dimensional partitions. The LMOV conjecture states that the expectation value of (2) takes the form

$$
\begin{aligned}
\langle Z(U, V)\rangle & =\sum_{R} \bar{P}_{R}(a, q) \operatorname{Tr}_{R} V \\
& =\exp \left(\sum_{n=1}^{\infty} \sum_{R} \frac{1}{n} f_{R}\left(a^{n}, q^{n}\right) \operatorname{Tr}_{R} V^{n}\right),
\end{aligned}
$$

where the expectation value of the holonomy is identified with the unreduced HOMFLY-PT polynomial of a knot $K,\left\langle\operatorname{Tr}_{R} U\right\rangle=\bar{P}_{R}(a, q)=P_{R}^{\mathbf{0}_{1}} P_{R}(a, q)$ (where $P_{R}^{\mathbf{0}_{1}}$ is the unknot factor), and the functions

$$
f_{R}(a, q)=\sum_{i, j} \frac{N_{R, i, j} a^{i} q^{j}}{q-q^{-1}}
$$

encode conjecturally integer LMOV (or Ooguri-Vafa) invariants $N_{R, i, j}$, counting bound states of M2-branes ending on M5-branes. These functions take the form of universal polynomials in colored HOMFLY-PT polynomials.

In what follows, we consider the one-dimensional source $V=x$. In this case, $\operatorname{Tr}_{R} V \neq 0$ only for symmetric representations $R=S^{r}$, so that $\operatorname{Tr}_{S^{r}}(x)=x^{r}$. Let us denote $P(x)=\langle Z(U, x)\rangle$, and let $\bar{P}_{r}(a, q)$ denote the $S^{r}$-colored HOMFLY-PT polynomial of $K$, so that (2) takes the form

$$
P(x)=\sum_{r=0}^{\infty} \bar{P}_{r}(a, q) x^{r}=e^{\sum_{r, n \geq 1} \frac{1}{n} f_{r}\left(a^{n}, q^{n}\right) x^{n r}} .
$$

In this case, $f_{r}(a, q) \equiv f_{S^{r}}(a, q)=\sum_{i, j} \frac{N_{r, i, j} a^{i} q^{j}}{q-q^{-1}}$, for $N_{r, i, j} \equiv$ $N_{S^{r}, i, j}$, are polynomials of $\bar{P}_{r / d_{1}}\left(a^{d_{2}}, q^{d_{2}}\right)$ for all divisors $d_{1}$ and $d_{2}$ of $r$, e.g., $f_{1}(a, q)=\bar{P}_{1}(a, q)$, 
BPS STATES, KNOTS, AND QUIVERS

$$
f_{2}(a, q)=\bar{P}_{2}(a, q)-\frac{1}{2} \bar{P}_{1}(a, q)^{2}-\frac{1}{2} \bar{P}_{1}\left(a^{2}, q^{2}\right),
$$

etc. It follows that (4) can be rewritten in product form

$$
P(x)=\prod_{r \geq 1 ; i, j ; k \geq 0}\left(1-x^{r} a^{i} q^{j+2 k+1}\right)^{N_{r, i, j}} .
$$

Integrality of BPS degeneracies $N_{r, i, j}$ encoded in this product is one important outcome of our work.

Another outcome of our work is a surprising relation of colored HOMFLY-PT polynomials, or their generating function (4), to superpolynomials, i.e., the Poincaré polynomials of the uncolored HOMFLY-PT homologies of knots [24]. More generally, one can consider colored HOMFLY-PT homology $\mathcal{H}_{i, j, k}^{S^{r}}$ that has not been defined rigorously by mathematicians, however, its conjectural (reduced) colored superpolynomial

$$
P_{r}(a, q, t)=\sum_{i, j, k} a^{i} q^{j} t^{k} \operatorname{dim} \mathcal{H}_{i, j, k}^{S^{r}}
$$

can be very effectively computed for various families of knots, using the formalism of differentials [24,25,27,28]. Note that generalizations of the LMOV conjecture to the case of superpolynomials have been considered in $[11,29]$.

For example, for trefoil knot $3_{1}$, Eq. (7) takes the form [30]

$P_{r}(a, q, t)=\frac{a^{2 r}}{q^{2 r}} \sum_{k=0}^{r}\left[\begin{array}{l}r \\ k\end{array}\right] q^{2 k(r+1)} t^{2 k} \prod_{i=1}^{k}\left(1+a^{2} q^{2(i-2)} t\right)$,

where $\left[\begin{array}{l}r \\ k\end{array}\right]=\frac{\left(q^{2} ; q^{2}\right)_{r}}{\left(q^{2} ; q^{2}\right)_{k}\left(q^{2} ; q^{2}\right)_{r-k}}$, and the q-Pochhammer symbol is defined as $(z ; q)_{n}=\prod_{i=0}^{n-1}\left(1-z q^{i}\right)$. For $t=-1$, (8) specializes to the reduced colored HOMFLY-PT polynomial, while in the uncolored $(r=1)$ case, it reduces to

$$
P_{1}(a, q, t)=\frac{a^{2}}{q^{2}}+a^{2} q^{2} t^{2}+a^{4} t^{3} .
$$

The monomials in this expression correspond to generators of the HOMFLY-PT homology, and powers of $t$ in each monomial—in this example, taking values $(0,2,3)$ - are referred to as homological degrees.

\section{MODULI OF QUIVER REPRESENTATIONS}

We now turn to a seemingly unrelated field of moduli of quiver representations. A quiver $Q$ is an oriented graph with a finite set of vertices $Q_{0}$ and a finite number of arrows between vertices $\alpha: i \rightarrow j$. On $\mathbf{Z} Q_{0}$, we define the Euler form of $Q$ by $\langle\boldsymbol{d}, \boldsymbol{e}\rangle_{Q}=\sum_{i \in Q_{0}} d_{i} e_{i}-\sum_{\alpha: i \rightarrow j} d_{i} e_{j}$. A quiver representation assigns to each vertex $i \in Q_{0}$ a vector space of dimension $d_{i}$ and a linear map to each arrow. Recently, it turned out that the structure of moduli spaces of quiver representations is very rich, and it is a natural playground for the theory of (motivic) Donaldson-Thomas invariants, cohomological Hall algebras, etc. In particular, various
PHYSICAL REVIEW D 96, 121902(R) (2017)

explicit results that we use in what follows are known for symmetric quivers, i.e., such that for any pair of vertices $i$ and $j$, the number of arrows from $i$ to $j$ equals the number of arrows from $j$ to $i$ [17,31-33].

Of our main focus in what follows will be the following motivic generating series assigned to a symmetric quiver

$$
P_{Q}(x)=\sum_{d \in \mathbf{N} Q_{0}}(-q)^{-\langle\boldsymbol{d}, \boldsymbol{d}\rangle_{Q}} \boldsymbol{x}^{d} \prod_{i \in Q_{0}} \prod_{j=1}^{d_{i}} \frac{1}{1-q^{-2 j}},
$$

where $\boldsymbol{x}^{\boldsymbol{d}}=\prod_{i \in Q_{0}} x_{i}^{d_{i}}$. Motivic Donaldson-Thomas invariants $\Omega_{\boldsymbol{d}, j} \equiv \boldsymbol{\Omega}_{d_{1}, \ldots, d_{m} ; j}$ (with $m$ denoting the number of vertices) are then defined via the factorization

$$
P_{Q}(x)=\prod_{d \neq 0} \prod_{j \in \mathbb{Z}} \prod_{k \geq 0}\left(1-x^{d} q^{j+2 k+1}\right)^{(-1)^{j+1} \Omega_{d, j}},
$$

and proved to be positive integers [31]. In [32,33], two geometric interpretations of coefficients of $\Omega_{\boldsymbol{d}}(q)$ are given: as the intersection Betti numbers of the moduli space of all semisimple representations of $Q$ of dimension vector $\boldsymbol{d}$, or as the Chow-Betti numbers of the moduli space of all simple representations of $Q$ of dimension vector $\boldsymbol{d}$.

\section{KNOT INVARIANTS FROM QUIVER REPRESENTATION THEORY}

We present now our main claim, which is the statement that various types of knot invariants, for a given knot, are encoded in the data of moduli spaces of quiver representations of a certain quiver, assigned to this knot. As explained above, from a physical perspective, this is a consequence of the supersymmetric quiver quantum mechanics description of BPS states in brane systems representing knots. In detail, this statement is a consequence of our observation that generating functions of colored HOMFLY-PT polynomials (4) can be written in the form

$P(x)=\sum_{d_{1}, \ldots, d_{m} \geq 0} q^{\sum_{i, j} C_{i, j} d_{i} d_{j}} \frac{\prod_{i=1}^{m} x^{d_{i}} q^{l_{i} d_{i}} a^{a_{i} d_{i}}(-1)^{t_{i} d_{i}}}{\prod_{i=1}^{m}\left(q^{2} ; q^{2}\right)_{d_{i}}}$

where $C$ is a symmetric $m \times m$ matrix, and $l_{i}, a_{i}$, and $t_{i}$ are fixed integers. We checked that colored polynomials can be rewritten in the form (12) in many examples, including all knots up to six crossings, infinite series of twist or torus knots, some knots with thick homology, etc., as will be reported in detail in forthcoming works [34]. Based on this ample evidence, we pose (12) as a general conjecture.

Remarkably, expression (12) has the same form as the motivic generating function (10) of a certain quiver, up to the identification $q \mapsto-q$ and the specialization

$$
x_{i}=x a^{a_{i}} q^{l_{i}-1}(-1)^{t_{i}} .
$$

In particular, terms proportional to $x^{r}$ in (12), with fixed $r$, arise from sets of $\left\{d_{i}\right\}$ such that $r=d_{1}+\cdots+d_{m}$. Rewriting of (4) in the form (12) means that the matrix 
KUCHARSKI, REINEKE, STOŠIĆ, and SUŁKOWSKI

$C$ can be identified as a matrix representing a quiver with $m$ vertices, such that $C_{i, j}$ denotes the number of arrows from vertex $i$ to $j$ (and so $C_{i, i}$ denotes the number of loops at vertex $i$ ). Therefore, if colored HOMFLY-PT polynomials for a given knot are known, after rewriting the generating function (4) in the form (12), from the form of $C$ the corresponding quiver can be identified. This also means that colored HOMFLY-PT polynomials for a given knot are encoded in a finite number of parameters that determine (12): the matrix $C$ and integers $l_{i}, a_{i}$, and $t_{i}$. Furthermore, these parameters take specific values, which are encoded in the uncolored superpolynomial. Recall that the uncolored, reduced superpolynomial for a given knot (such as (9) for the trefoil) is a sum of monomials of the form $a^{a_{i}} q^{q_{i}} t^{t_{i}}$, which correspond to generators of the HOMFLY-PT homology. We claim that the size of the matrix $C$ (and so the number of vertices in the corresponding quiver) is equal to the number of such generators. Moreover, with appropriate ordering of vertices, $t_{i}$ in (12) are to be identified with homological degrees of generators of HOMFLY-PT homology, diagonal elements of $C$ are also equal to homological degrees, i.e., $C_{i, i}=t_{i}$, coefficients of linear powers of $q$ take the form $l_{i}=q_{i}-t_{i}$, and $a_{i}$ are equal to $a$ degree of generators of uncolored HOMFLY homology. An additional minus sign in (12) comes with the power determined by $t_{i}$, so that it is relevant only for the generators with odd $t$ grading. Note that it follows that homological degrees $t_{i}$ can be read off from the generating series (4) rewritten in the quiverlike form, i.e., they are given by the number of loops in the corresponding quiver; this means that the uncolored superpolynomial is encoded in the form of colored HOMFLY-PT polynomials, which is a rather nontrivial and previously unknown statement.

Note that the values of parameters $l_{i}, a_{i}$, and $C_{i, i}$ depend in fact on a choice of normalization of $\bar{P}_{r}(a, q)$. The values mentioned above, related to the unreduced and uncolored superpolynomial, arise when normalization includes only the denominator of the colored HOMFLY-PT polynomial of the unknot $\bar{P}_{r}(a, q)=P_{r}(a, q) /\left(q^{2} ; q^{2}\right)_{r}$. Perhaps more familiar normalization by the full unknot polynomial $\bar{P}_{r}(a, q)=a^{-r} q^{r} \frac{\left(a^{2} ; q^{2}\right)_{r}}{\left(q^{2} ; q^{2}\right)_{r}} P_{r}(a, q)$ leads to identification with another version of HOMFLY-PT homology and a quiver of twice the size [34].

The above conjecture has very nontrivial consequences. In general, it implies that various knot invariants are specializations of certain quiver moduli invariants. In particular, under the above specialization, the product decomposition (11) is identified with the product decomposition (6). It follows that LMOV invariants $N_{r, i, j}$ can be expressed as linear combinations (with integer coefficients) of motivic Donaldson-Thomas invariants $\Omega_{\boldsymbol{d}, j} \equiv \Omega_{d_{1}, \ldots, d_{m} ; j}$. As motivic Donaldson-Thomas
PHYSICAL REVIEW D 96, 121902(R) (2017)

invariants are proven to be integer, it follows that corresponding LMOV invariants are also integer, which proves the LMOV conjecture. We are able to prove this statement for many specific knots (and arbitrary symmetric representations), including some infinite families of knots (e.g., twist knots, certain classes of torus knots), analogously as in the case of trefoil knot, which is analyzed in detail below. Moreover, the limit $q \rightarrow 1$ of the motivic generating series immediately implies integrality of classical LMOV invariants $b_{r, i}=\sum_{j} N_{r, i, j}$ (considered, for example, in [11]), which are then expressed in terms of (integer) numerical DonaldsonThomas invariants. Moreover, the fact that LMOV invariants and (generating functions of) colored HOMFLY-PT polynomials are expressed in terms of motivic Donaldson-Thomas invariants-which arise as certain Betti numbers of quiver moduli spaces-provides a novel categorification of these knot invariants.

Some other relations between knots and quivers that follow from our conjecture are listed in Table I. For example, the framing by $f \in \mathbb{Z}$ changes the colored HOMFLY-PT polynomial by a factor, that for $S^{r}$-symmetric representation takes form $a^{2 f r} q^{r(r-1)}$. The term with quadratic power of $q$, i.e., $q^{f r^{2}}=q^{f\left(\sum_{i} d_{i}\right)^{2}}=q^{f \sum_{i, j} d_{i} d_{j}}$ shifts all elements of $C$ by $f$, which in the dual quiver adds $f$ loops at each vertex and $f$ pairs of oppositely oriented arrows between all pairs of vertices. Moreover, as predicted in [17], the cohomological Hall algebra associated with a quiver should be identified as the algebra of BPS states [16], which deserves further studies in the context of brane realization of knot invariants.

\section{EXAMPLE: TREFOIL KNOT}

We illustrate the above correspondence in the example of the trefoil knot, whose reduced colored HOMFLYPT polynomials arise by setting $t=-1$ in (8). Using the $q$-binomial identity $(z ; q)_{k}=\sum_{i}\left[\begin{array}{l}k \\ i\end{array}\right](-z)^{i} q^{\frac{i(i-1)}{2}}$, the $q$ binomial and the last product in (8) take the form

$$
\left[\begin{array}{l}
r \\
k
\end{array}\right]\left(\frac{a^{2}}{q^{2}} ; q^{2}\right)_{k}=\sum_{i=0}^{k} \frac{\left(q^{2} ; q^{2}\right)_{r}\left(-\frac{a^{2}}{q^{2}}\right)^{i} q^{i(i-1)}}{\left(q^{2} ; q^{2}\right)_{r-k}\left(q^{2} ; q^{2}\right)_{i}\left(q^{2} ; q^{2}\right)_{k-i}} .
$$

Introducing $r=d_{1}+d_{2}+d_{3}, k=d_{2}+d_{3}, i=d_{3}$ with $d_{i} \geq 0$, and normalizing $P_{r}(a, q)$ by $\left(q^{2} ; q^{2}\right)_{r}$, the generating function (4) can be rewritten as in (12)

$$
\begin{aligned}
P(x) & =\sum_{r=0}^{\infty} \frac{P_{r}(a, q)}{\left(q^{2} ; q^{2}\right)_{r}} x^{r} \\
& =\sum_{d_{1}, d_{2}, d_{3} \geq 0} \frac{q^{\sum_{i, j} C_{i, j} d_{i} d_{j}-2 d_{1}-3 d_{3}}(-1)^{d_{3}} a^{2 d_{1}+2 d_{2}+4 d_{3}} x \sum_{i} d_{i}}{\left(q^{2} ; q^{2}\right)_{d_{1}}\left(q^{2} ; q^{2}\right)_{d_{2}}\left(q^{2} ; q^{2}\right)_{d_{3}}}
\end{aligned}
$$


with

$$
C=\left[\begin{array}{lll}
0 & 1 & 1 \\
1 & 2 & 2 \\
1 & 2 & 3
\end{array}\right]
$$

The corresponding quiver is shown in Fig. 1 and, as we claimed above, its vertices correspond to generators of HOMFLY-PT homology. Furthermore, diagonal elements $(0,2,3)$ of matrix $C$ (representing numbers of loops at vertices of the quiver) indeed agree with homological degrees encoded in the uncolored superpolynomial (9), coefficients $l_{i}=-2,0$, 3 of linear terms in $d_{i}$ in the power of $q$ in (14) are given by $l_{i}=q_{i}-t_{i}$, coefficients $a_{i}=2,2,4$ in the power of $a$ agree with $a$ degrees of generators of HOMFLY-PT homology, and the additional minus sign $(-1)^{d_{3}}$ is determined by just one generator with odd $t$ degree $t_{3}=3$.

We stress that rewriting of the generating function of colored HOMFLY-PT polynomials in the form (14) guarantees that it can be written in a product form (11), so that LMOV invariants are expressed as combinations (with integer coefficients) of integer motivic DonaldsonThomas invariants $\Omega_{d_{1}, d_{2}, d_{3} ; j}$. This proves the LMOV conjecture for the $3_{1} \mathrm{knot}$, for all symmetric representations.

As mentioned below (12), analogous calculations can be done for many other knots, e.g., rewriting formulas from [30]. A general formalism facilitating such computations and their consequences will be presented in [34].

\section{SUMMARY}

In this paper, we presented a surprising duality between knots and quivers, which underlies the supersymmetric quiver quantum mechanics description of BPS states in brane systems describing knots. To conclude, let us indicate various directions in which relations presented above could be generalized. It is natural to expect that various properties, well known on one side of the duality, should have their counterparts on the other side.

First, it is desirable to be able to identify a quiver corresponding to a given knot more directly, not necessarily taking advantage of colored HOMFLY-PT polynomials, on which the method presented above relies.
Second, colored HOMFLY-PT polynomials and LMOV invariants are naturally defined for arbitrary representations, while for symmetric representations they are related to algebraic curves of $A$-polynomial type, as well as recursion relations encoded in $\hat{A}$ polynomials $[11,12,35]$. We expect that analogous objects should exist for quivers, presumably generalizing observations in [18].

On the other hand, various properties of quivers, their moduli spaces, and other related objects - in particular, cohomological Hall algebra-should shed new light on properties of knot invariants, in particular, knot homologies. Further, from the quiver representation viewpoint, it is natural to split the generating parameter $x$ into parameters $x_{1}, \ldots, x_{m}$, which implies a nontrivial refinement of LMOV invariants (with a label $d$ split into $d_{1}, \ldots, d_{m}$ ), as in (11), as well as of HOMFLY-PT polynomials.

It is also interesting to relate our results to other developments, e.g., to the connection-albeit in a different context-between (uncolored) HOMFLY-PT polynomials and Donaldson-Thomas invariants in [36]. Then, the generating functions (12) take the form of combinations of $q$-series that appear in Nahm's conjecture [37], which indicates their relation to integrable systems and conformal field theory.

We believe that the results and conjectures presented in this paper are just a tip of the iceberg, which is worth thorough exploration.

\section{ACKNOWLEDGMENTS}

We thank Sergei Gukov, Satoshi Nawata, Miłosz Panfil, Yan Soibelman, Richard Thomas, Cumrun Vafa, and Paul Wedrich for discussions and comments on the manuscript. This work is supported by the ERC Starting Grant No. 335739 "Quantum fields and knot homologies” funded by the European Research Council under the European Union's Seventh Framework Programme, and the Foundation for Polish Science. M. S. is partially supported by the Ministry of Science of Serbia, Project No. 174012, and by Fundação para a Ciência e a Tecnologia (FCT), through the FCT Investigador Grant.
[1] R. Gopakumar and C. Vafa, arXiv:hep-th/9812127.

[2] D. Maulik, N. Nekrasov, A. Okounkov, and R. Pandharipande, Compos. Math. 142, 1263 (2006).

[3] K. Hori, S. Katz, A. Klemm, R. Pandharipande, R. Thomas, C. Vafa, R. Vakil, and E. Zaslow, Mirror Symmetry, Clay Mathematics Monographs Vol. 1 (American Mathematical Society, Providence, 2003).

[4] H. Ooguri and C. Vafa, Nucl. Phys. B577, 419 (2000).
[5] J. M. F. Labastida and M. Marino, Commun. Math. Phys. 217, 423 (2001).

[6] J. M. F. Labastida, M. Marino, and C. Vafa, J. High Energy Phys. 11 (2000) 007.

[7] E. Witten, Commun. Math. Phys. 121, 351 (1989).

[8] E. Witten, Prog. Math. 133, 637 (1995).

[9] P. Ramadevi and T. Sarkar, Nucl. Phys. B600, 487 (2001). 
KUCHARSKI, REINEKE, STOŠIĆ, and SUŁKOWSKI

[10] A. Mironov, A. Morozov, A. Morozov, P. Ramadevi, V. K. Singh, and A. Sleptsov, J. High Energy Phys. 08 (2017) 139.

[11] S. Garoufalidis, P. Kucharski, and P. Sulkowski, Commun. Math. Phys. 346, 75 (2016).

[12] P. Kucharski and P. Sulkowski, J. High Energy Phys. 11 (2016) 120.

[13] K. Liu and P. Peng, J. Diff. Geom. 85, 479 (2010).

[14] F. Denef, J. High Energy Phys. 10 (2002) 023.

[15] M. Alim, S. Cecotti, C. Cordova, S. Espahbodi, A. Rastogi, and C. Vafa, Adv. Theor. Math. Phys. 18, 27 (2014).

[16] J. A. Harvey and G. W. Moore, Commun. Math. Phys. 197, 489 (1998).

[17] M. Kontsevich and Y. Soibelman, Commun. Number Theory 5, 231 (2011).

[18] M. Reineke, Compos. Math. 147, 943 (2011).

[19] M. Reineke, Doc. Math. 17, 1 (2012).

[20] M. Khovanov, Duke Math. J. 101, 359 (2000).

[21] M. Khovanov and L. Rozansky, arXiv:math/0401268.

[22] M. Khovanov and L. Rozansky, Geom. Topol. 12, 1387 (2008).

[23] S. Gukov, A. Schwarz, and C. Vafa, Lett. Math. Phys. 74, 53 (2005).
PHYSICAL REVIEW D 96, 121902(R) (2017)

[24] N. M. Dunfield, S. Gukov, and J. Rasmussen, Exp. Math. 15, 129 (2006).

[25] S. Gukov and M. Stosic, Proc. Symp. Pure Math. 85, 125 (2012).

[26] J. M. F. Labastida and M. Marino, J. Knot Theory Ramif. 11, 173 (2002).

[27] J. Rasmussen, Geom. Topol. 19, 3031 (2010).

[28] S. Gukov, S. Nawata, I. Saberi, M. Stosic, and P. Sulkowski, J. High Energy Phys. 03 (2016) 004.

[29] M. Kameyama and S. Nawata, arXiv:1703.05408.

[30] H. Fuji, S. Gukov, P. Sulkowski, and M. Stosic, J. High Energy Phys. 01 (2013) 175.

[31] A. I. Efimov, Compos. Math. 148, 1133 (2012).

[32] H. Franzen and M. Reineke, arXiv:1512.03748.

[33] S. Meinhardt and M. Reineke, arXiv:1411.4062.

[34] P. Kucharski, M. Reineke, M. Stosic, and P. Sulkowski, arXiv:1707.04017.

[35] M. Aganagic and C. Vafa, arXiv:1204.4709.

[36] D.-E. Diaconescu, Z. Hua, and Y. Soibelman, Commun. Number Theory 6, 517 (2012).

[37] W. Nahm, Proceedings of the Frontiers in Number Theory, Physics, and Geometry 2: On Random Matrices, Zeta Functions and Dynamical Systems, Les Houches, France, 2003, arXiv:hep-th/0404120. 